\title{
Advances in weight loss surgery and body contouring after weight loss
}

\author{
Charles K Herman ${ }^{1,2}$ \\ Ari S Hoschander ${ }^{3}$ \\ Nicolas Teleo' \\ Berish Strauch ${ }^{2}$
}

'Pocono Medical Center/Pocono Health System, East Stroudsburg, PA, ${ }^{2}$ Albert Einstein College of Medicine, New York, NY; ${ }^{3}$ Northshore Long Island Jewish Health System, Manhasset, NY, USA
Correspondence: Charles K Herman 100 Plaza Court, Suite C, East Stroudsburg, PA I830I, USA

$\mathrm{Tel}+\mathrm{I} 5704206220$

Fax + I 570420622 I

Email charleshermanmd@hotmail.com
This article was published in the following Dove Press journal:

Open Access Surgery

24 June 2010

Number of times this article has been viewed
Abstract: Surgical treatment for morbid obesity has been increasing over the last few years. Historically, bariatric surgery had been plagued by complications, both technical and metabolic. The criteria to decide if a patient should have bariatric surgery required that the patient's morbid obesity was contributing to an otherwise imminent demise. Now, with improved techniques, laparoscopic surgery and better understanding of the metabolic consequences of bariatric surgeries, it is presumed that the benefits outweigh the risks of bariatric surgery in increasing numbers of patients. Additionally, the post-bariatric procedures for body contouring have many documented complications. Most of these can be related to the nutritional status of obese patients and the relative immunodeficiency that obese patients possess. After a thorough literature search it appears that bariatric surgery and post-bariatric body contouring are, indeed, safer than previously thought.

Keywords: body contouring, gastric bypass, bariatric surgery

\section{Introduction}

Obesity is a serious disease that carries substantial morbidity and mortality and has mixed genetic and environmental etiologies. Obesity is defined as the accumulation of excess body fat that leads to pathology. Severity is based on the degree of excess body fat, and is commonly assessed using the body mass index (BMI) which consists of body weight divided by height in meters squared. Obesity may be defined as body weight that exceeds ideal body weight by $20 \%$ or BMI greater than $30 \mathrm{~kg} / \mathrm{m}^{2}$. Morbidly obese individuals generally exceed ideal body weight by 100 pounds or more, or are $100 \%$ over their ideal body weight. In 1991, the US National Institute of Health defined morbid obesity as a BMI of $35 \mathrm{~kg} / \mathrm{m}^{2}$ or greater with comorbidities, or BMI of $40 \mathrm{~kg} / \mathrm{m}^{2}$ or greater without a comorbidity. Superobesity is a term sometimes used to define individuals who have body weight exceeding ideal body weight by $225 \%$ or more, or a BMI of $50 \mathrm{~kg} / \mathrm{m}^{2}$ or greater.

Bariatric surgery, when successful, leaves the patient with the sequelae of massive weight loss and of the bariatric operation itself. The functional problems associated with massive weight loss are usually related to skin and fascia excess or relaxation. Redundant skin primarily causes problems that include intertrigo, pain, problems maintaining appropriate hygiene, and decreased activity. ${ }^{1}$ After massive amounts of weight loss, the patient is often unable to begin a proper exercise regimen, an important step in the recovery from obesity, secondary to the excess pendulous tissue. Friction between the thighs can also cause pain and discomfort when ambulating. Massive 
weight loss patients who have undergone bariatric surgery have also been shown to have a higher risk of postoperative incisional hernias. These hernias may cause both functional and aesthetic problems. Reported rates of incisional hernia following open gastric bypass are as high as $41 \%{ }^{2}$ The hernia itself can cause significant functional disability in addition to the cosmetic deformity, including pain, discomfort with movement, and impairment of mobility. In some instances this can restrict patients from adequately performing normal daily activities and possibly prevent them from attending work. ${ }^{3}$ Body contouring and incisional hernia repair have become an integral part of the care for post-bariatric surgery patients.

Bariatic and post-bariatric body contouring procedures have undergone a profound evolution over the past two decades. Due to the advent of minimally-invasive bariatric techniques, a greater understanding of the anatomy and physiology of the bariatric surgery patient, and refinement in body contouring techniques, these procedures are now demonstrating lower than ever risk to benefit with a high margin of safety. We review historic and modern techniques utilized in bariatric and body contouring surgery as well as the outcomes data available, including complications, morbidity and mortality, and patient satisfaction.

\section{Bariatric surgery: goals and mechanisms of action}

The goal of bariatric surgery is to improve health in morbidly obese patients by achieving long-term, durable weight loss. It involves reducing caloric intake and/or absorption of calories from food, and it may modify eating behavior by promoting slow ingestion of small boluses of food.

Restrictive operations restrict the amount of food intake by rationing the quantity of food that can be consumed at one time, which results in a reduction in caloric intake. Malabsorptive procedures limit the absorption of nutrients and calories from ingested food by limiting absorption to the duodenum and predetermined lengths of small intestine.

The operations currently in use for the management of morbid obesity involve gastric restriction with or without intestinal malabsorption. Gastric restrictive procedures include laparoscopic vertical banded gastroplasty (LVBG) and laparoscopic adjustable gastric banding (LAGB). Malabsorptive procedures include biliopancreatic diversion (BPD), and biliopancreatic diversion with duodenal switch (BPD-DS). Roux-en-Y gastric bypass has features of both restriction and malabsorption. The advent of laparoscopic techniques has allowed surgeons to offer minimally-invasive approaches to these bariatric procedures.

\section{Bariatric surgical procedures and outcomes}

The VBG is purely restrictive in nature, limiting the amount of solid food that can be consumed at one time, which leads to a caloric deficit. This procedure has become less commonly used recently. A proximal gastric pouch empties through a calibrated stoma, which is reinforced by a strip of mesh or an elastic ring. VBG has been shown to achieve acceptable weight loss results in obese patients. A study by van de Weijgert et $\mathrm{al}^{4}$ demonstrated a mean excess weight loss of $63 \%$ after 7 years in $100 \mathrm{VBG}$ patients. With weight loss following VBG, there is a significant improvement in comorbidities, including dyspnea, hypertension, diabetes mellitus, orthopedic problems, and quality of life. VBG is associated with only minimal long-term metabolic or nutritional deficiencies. Long-term weight loss is less successful if patients ingest large amounts of sweets or high-calorie liquids. A Cochrane review, ${ }^{5}$ analyzing the different types of gastric restrictive and malabsorptive procedures, concluded that VBG has a low complication rate with a mortality of $1 \%$ and a reoperation and revision rate of $30 \%$.

Laparoscopic adjustable gastric banding involves placement of a silicone band around the proximal stomach to restrict the amount of solid food that can be ingested at one time. Furthermore, the adjustable nature of the band allows the amount of restriction to be increased or decreased, depending on the patient's weight loss. Suter et $\mathrm{l}^{6}$ published their results with LAGB after 3 years of experience. One hundred and fifty patients underwent laparoscopic gastric banding with mean body mass index of $44.6 \mathrm{~kg} / \mathrm{m}^{2}$ (range from 35.1 to $64.1 \mathrm{~kg} / \mathrm{m}^{2}$ ) and mean initial excess body weight of $102.9 \%$. Mean follow-up was 17 months and mean excess weight loss at 1 and 2 years was 55 and $56 \%$, respectively. LAGB is considered to be the safest and least invasive of all of the surgical weight loss procedures. Its reversibility gives it an additional level of safety. The dreaded complication of gastric band erosion into the stomach occurs infrequently in less than $2 \%$ of cases. ${ }^{7}$ Additional complications include intraoperative injuries, such as splenic and esophageal injuries, and postoperative complications including band slippage and wound problems. In a randomized trial utilizing the gastric band in severely obese adolescents, O'Brien et $\mathrm{al}^{8}$ found that patients in the banding group lost an average of almost $35 \mathrm{~kg}$ for a mean excess weight loss of nearly $80 \%$. 
This group had no perioperative adverse events although they did have a reoperation rate of $33 \%$ for band revision. Quality of life and health were improved in this group of formerly obese adolescents.

Laparoscopic RYGB is both a gastric restrictive procedure and a malabsorptive procedure. A small gastric pouch restricts food intake, while the Roux-en-Y configuration provides malabsorption of calories and nutrients. Most comorbidities were improved or eradicated, including diabetic mellitus, hypertension, sleep apnea, and gastric reflux. A recent analysis of 500 patients who underwent RYGB had a complication rate of only $13 \% .{ }^{9}$ This study had no mortalities and the most frequent complications included infection, leak, respiratory problems, stenosis of one of the anastomoses, small bowel obstruction, and hemorrhage. Additionally, the authors noted that these complications occurred with significantly less frequency after the first 300 operations were performed.

\section{Post-bariatric body contouring procedures and outcomes}

It is important to recognize that massive weight loss patients have complex problems that span multiple parts of the body. Presurgical planning should be extensive. A comprehensive history and physical should be performed. The history should include, but not be limited to, comorbidities such as coronary artery disease, hypertension, diabetes, and history of smoking. A physical examination should be performed with special attention being paid to areas with the most skin redundancy and areas with the worst objective sequelae. The presence of an incisional hernia should be noted and assessed for size. Some plastic and reconstructive surgeons will consult with a general surgeon preoperatively for patients requiring hernia repair, while others are comfortable performing the hernia repair themselves. Preoperative labs and medical or cardiac clearance should also be obtained. The proper timing of the surgery is after the patient's weight loss has stabilized and after the patient's body mass index is no longer in the obese range. A recent study found that $\mathrm{BMI}>30$ at the time of surgery is correlated with a higher risk of complications. ${ }^{10}$ As with any complex surgery, an extensive preoperative work-up will benefit both the surgeon and the patient.

The discussion of safety in post-bariatric body contouring can be divided into complications that occur during individual procedures as well as those that occur during combinations of those procedures. A recent retrospective analysis by Shermak et al of the outcomes of patients who had body contouring procedures after massive weight loss found that $37 \%$ of those patients incurred at least one complication. ${ }^{11}$ The procedures performed on these patients included abdominal procedures as well as procedures on the arms, back, and chest. The authors found wound healing complications in $14.4 \%$ and seromas in $12.9 \%$. Less common complications included thromboembolic complications in $2.9 \%$, infections in $2.9 \%$, deep suture abscesses in $1.4 \%$, and postoperative bleeding in $1.4 \%$; drain removal under anesthesia, hematoma and lymphocele in the arm, pain requiring consultation, and a bleeding ulcer requiring transfusion occurred in $<1 \%$.

A retrospective study observing complications and their relationship to preoperative BMI found the total complication rate after panniculectomy to be $40 \%{ }^{12}$ Similar to the previously mentioned study, the most frequent complications included seroma in $17 \%$, surgical site infections in $17 \%$, and hematoma in $13 \%$ of patients. Additional complications included skin breakdown or necrosis in $11 \%$, reexploration in $11 \%$, and transfusion requirement in $6 \%$ of patients. Multiple variables in this group were analyzed with reference to complications: BMI was the only variable found to positively correlate with increased risk of complications.

Abdominal contouring in massive weight loss patients commonly involves circumferential skin excision. This procedure has been referred to in multiple ways by different authors. These names include circumferential abdominoplasty, belt lipectomy, and body lift. A recent paper by Strauch et al studied 75 consecutive mid-body lift procedures over five years. ${ }^{1}$ They found a total complication rate of $15 \%$. The majority of complications were related to wound healing, with $7 \%$ of patients experiencing wound edge necrosis with eventual healing by secondary intention. Scars requiring revision occurred in $2.9 \%$ of patients. The remaining complications occurred in only $1(1.4 \%)$ patient each and included epidermolysis, contour deformity requiring liposuction, seroma, and superficial thrombophlebitis. There were no thromboembolic events nor perioperative mortalities. Capella et al reviewed 200 consecutive body lifts and found the total complication rate to be $50 \% .{ }^{13}$ As with the aforementioned studies, the most frequently encountered complications were related to the wounds and the presence of seromas. The authors noted wound dehiscence in $32.5 \%$, seroma in $16.5 \%$, and skin necrosis in $9.5 \%$ of patients. Blood transfusion was required in $15.5 \%$ of patients. Infection, thromboembolic events and bleeding each occurred in less than $4 \%$ of all patients. It should be noted that the complication rates in smokers were significantly greater than in nonsmokers. Higher BMI was once again found to 
correlate with increased risk for complications in addition to increased length of stay. Figure 1 illustrates commonly used markings in lower body lifting.

Incisional hernia is a common complication from any open surgery and is considered to be the most commonly encountered technical problem following open gastric bypass. ${ }^{14}$ The indication to perform an incisional hernia repair is the presence of the hernia itself. It is often simpler to perform an incisional hernia repair at the time of abdominal contouring secondary to the added exposure of the fascia afforded by the abdominal wall flaps that have been created for adequate skin excision. The complications associated with abdominoplasty with concurrent hernia repair have been reported by Shermak et $\mathrm{al}^{15}$ who found them to be similar to those that occur when the two procedures are performed separately. These included problems with wound healing and seromas in $20 \%$ and $12.5 \%$ respectively. Suture abscess, bleeding, and cellulitis were also seen, as well as a fatal pulmonary embolism and a recurrent hernia, each occurring in 1 patient. Overall, this review showed the combination procedure to be both safe and effective. The authors frequently combine hernia repair at the time of body contouring. Components separation closure of hernia defects has been particularly useful in difficult hernias, as the abdominal fascia is commonly found to be lax and redundant in these cases, facilitating the advancement of muscle flaps (Figure 2).

Truncal contouring is the most common procedure after massive weight loss. However, the other frequently addressed body areas are the breasts, arms, and thighs. These procedures are often performed at the same time or in conjunction with the abdominal operation. Occasionally,
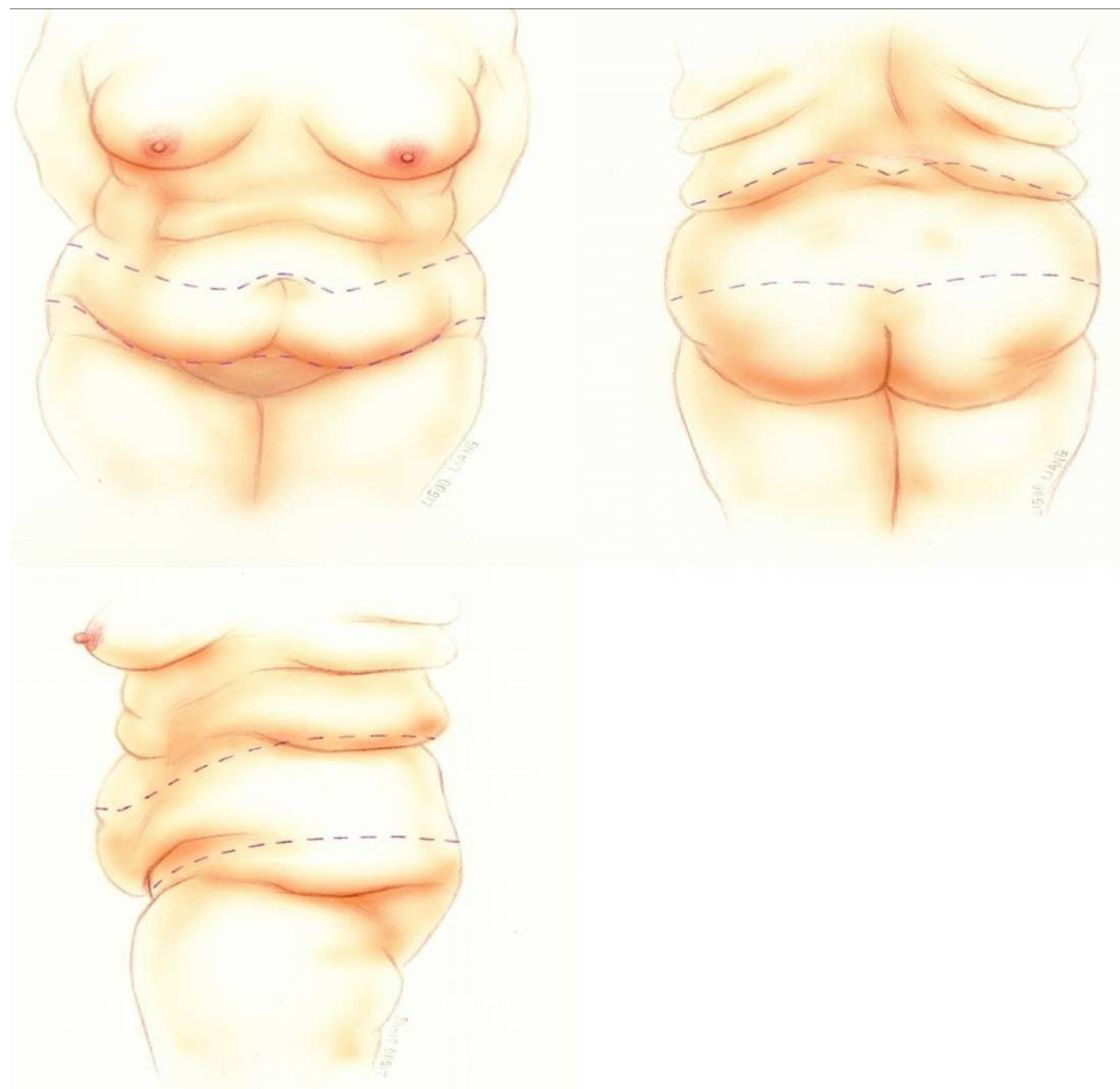

Figure I Preoperative markings for lower body lifting. 


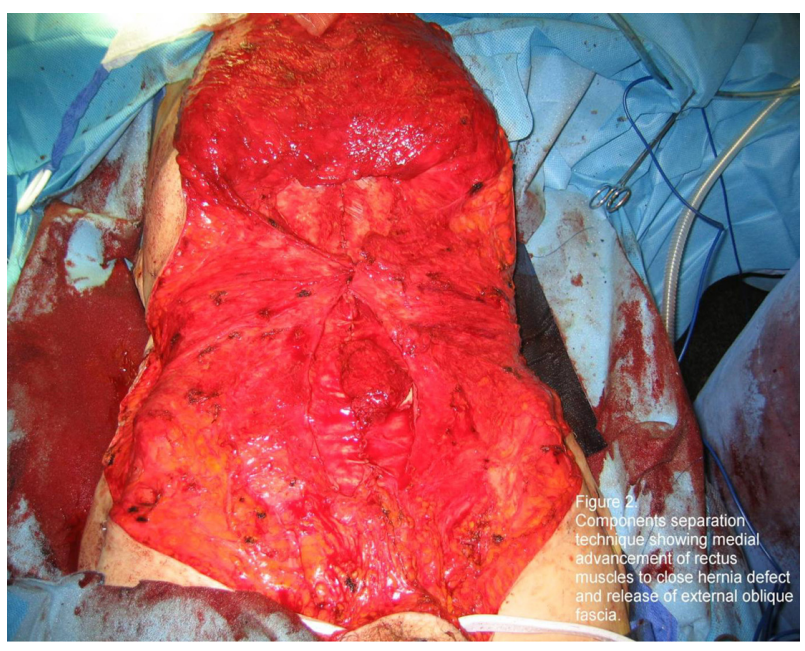

Figure 2 Components separation for closure of a ventral hernia.

3 body areas are addressed at the same time. Brachioplasty surgery was performed by Rubin et al in 101 patients after massive weight loss, among which $96 \%$ had a concomitant procedure. ${ }^{16}$ Complications specific to the brachioplasty procedures in their series occurred in $40.4 \%$. Of those, $71 \%$ were seromas which occurred with even more frequency in patients who had received arm liposuction. The remaining complications included dehiscence and wound infections. Figure 3 demonstrates preoperative and postoperative results after a lower body lift.

Mastopexy is another frequently performed operation after massive weight loss. Breast deformities after massive weight loss often include severe ptosis and the appearance of a 'deflated' breast. There are multiple types of operations that correct these deformities and they often have a high level of patient satisfaction. Rubin et al performed 108 consecutive mastopexy operations and found the operation to have a very low complication rate. ${ }^{17}$ The complications they encountered included seroma, dehiscence, and skin flap necrosis. These complications occurred in only 8 patients for a total of $7.5 \%$. This group also performed multiple different combinations of procedures at the time of the mastopexy including upper and lower body lifts, abdominoplasty, brachioplasty, and vertical medial thighplasty. The complications from these other procedures were significantly greater, with a total complication rate, including breast complications, of $52 \%$. Losken and Holtz attempted to determine patient satisfaction after mastopexy following massive weight loss, among other outcomes. ${ }^{18}$ Patient satisfaction was assessed utilizing a series of parameters. Twenty patients were asked to rate their results from 1 to $5(1=$ poor and $5=$ excellent $)$. The average result was 4.1 and $90 \%$ of these patients rated

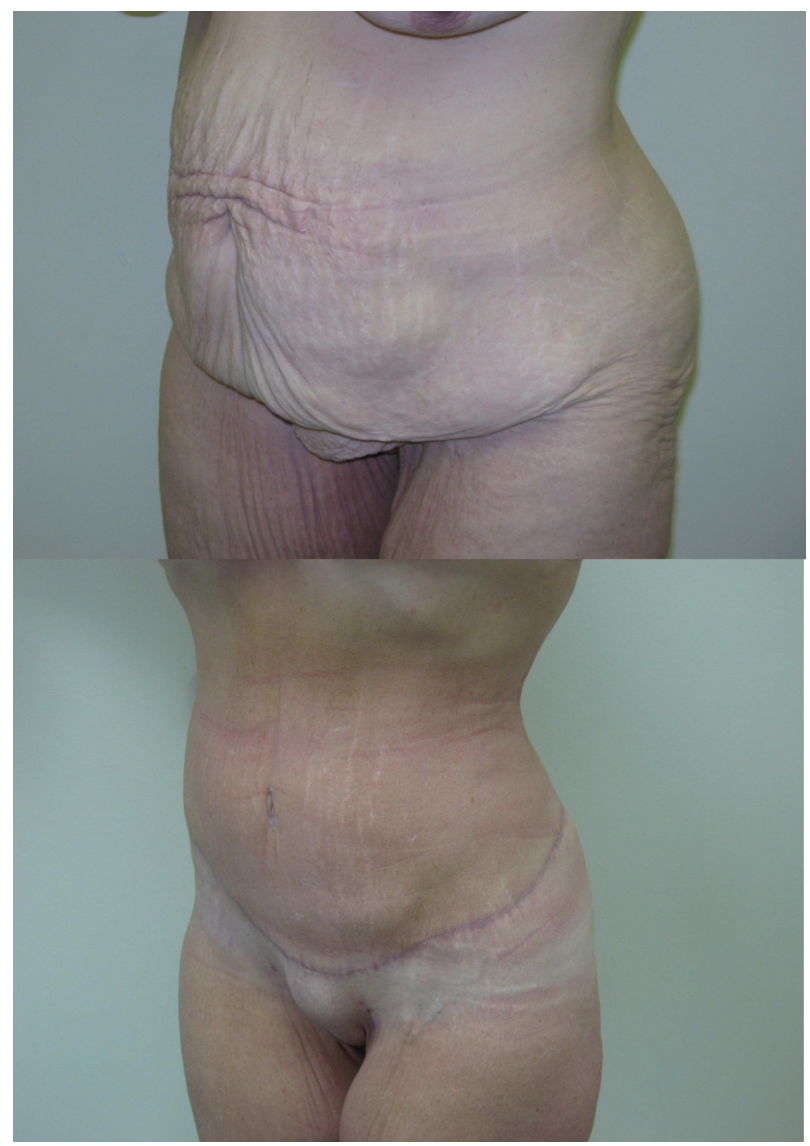

Figure 3 Preoperative and postoperative photographs after lower body lift.

their results 3 to 5 . Additionally, a total of 35 patients who underwent mastopexy in this series were found to have a total complication rate of $17 \%$. Complications included wound dehiscence, hypertrophic scarring, wound infection, and capsular contracture. Overall, mastopexy has been shown to be safe and to have a high level of satisfaction.

Medial thigh lifts are often performed and should be considered for patients with difficulty ambulating or with excessive friction between the thighs after massive weight loss. Additionally, the inability to maintain proper hygiene in this intertriginous area is a good indication for thigh reduction surgery. Currently, there is a paucity of literature related to the safety and complications attributable to this procedure. Most of the medial thigh lift studies contain only a small number of patients. Thigh lift surgery was performed by Bruschi et al in 35 patients, where noted complications included anemia in $43 \%$ and hematoma in $14 \% .^{19}$ Ellabban performed 14 medial vertical thigh reductions in massive weight loss patients and encountered postoperative thigh swelling in 4 patients. ${ }^{20}$ The authors attributed this to the sacrifice of the saphenous vein. They noted that after the procedure was tailored to avoid this sacrifice, thigh swelling 
no longer occurred. One of these 4 patients was also found to have an infected seroma. Shermak investigated complications following body contouring procedures and reported seroma occurrence in $4 \%$ of patients following thigh lift. ${ }^{21}$

\section{Discussion}

Bariatric surgery is currently considered to be safe and extremely effective. A meta-analysis by Buchwald et $\mathrm{al}^{22}$ concluded that bariatric surgery not only improves weight loss significantly in morbidly obese individuals, but also improves diabetes, hyperlipidemia, hypertension, and obstructive sleep apnea. Many of these patients have complete resolution of these comorbidities. In a preliminary analysis of the outcomes of bariatric surgery at hospitals that have been credited as centers of excellence, the early data show a significantly lower complication rate than previous reports have shown. ${ }^{23}$ The major adverse outcomes investigated included hospital mortality, operative mortality at 30 and 90 days, reoperation rates, and readmission. Overall mortality occurred in $0.36 \%$ of patients, with reoperation being necessary in $2 \%$ and readmission in $5 \%$ of patients. These values once again show that increased experience with these complicated procedures improves outcomes.

The care of formerly obese and super-obese patients should not end with weight reduction or weight reduction surgery. These patients still suffer from objective sequelae of their former disease. Body contouring surgery is a necessary adjunct to massive weight loss in order to restore the patient to a more functional state with a higher quality of life. These procedures include contouring of the abdomen and circumferential midsection, breasts, arms, and thighs. While there have been a high percentage of complications listed in this article, a vast majority of those complications were related to wound healing and seromas, most often not requiring any further interventions. We have found in our review of the literature and our own surgical experience that the benefits of post-bariatric contouring far outweigh the complications of the surgeries.

Our approach to post-bariatric body contouring is often multistaged and takes into the account the lessons learned from experience and from the literature. Complications can be reduced by limiting anesthesia time and staging the operations. Abdominoplasty or body lift is usually the first surgery performed. Breast and arm contouring are frequently performed together during the next stage, followed by thigh lifting. Although often requiring two to three stages over several months, patients often find their weight loss journey to be complete and satisfying after successful body contouring.

\section{Conclusion}

Taken together, data garnered from the literature and from our own experience demonstrate that complications from both bariatric procedures and the post-bariatric body contouring procedures are acceptable when compared to the benefits from these procedures. The objective reductions in weight loss and comorbidities, as well as the improvements in lifestyle, have proven to be extremely advantageous to these individuals who can now have a new life and a new lease on life.

\section{Disclosure}

The authors report no conflicts of interest in this work

\section{References}

1. Strauch B, Herman C, Rohde C, Baum T. Mid-body contouring in the post-bariatric surgery patient. Plast Reconstr Surg. 2006;117(7): 2200-2211.

2. Acarturk TO, Wachtman G, Heil B, Landecker A, Courcoulas AP, Manders EK. Panniculectomy as an adjuvant to bariatric surgery. Ann Plast Surg. 2004;53(4):360-366.

3. Millikan, KW. Incisional hernia repair. Surg Clin North Am. 2003;83(5):1223-1234.

4. van de Weijgert EJ, Ruseler CH, Elte JW. Long-term follow-up after gastric surgery for morbid obesity: preoperative weight loss improves the long-term control of morbid obesity after vertical banded gastroplasty. Obes Surg. 1999;9(5):426-432.

5. Colquitt JL, Picot J, Loveman E, Clegg AJ. Surgery for obesity. Cochrane Database Syst Rev. 2009;(2):CD003641.

6. Suter M, Bettschart V, Giusti V, Heraief E, Jayet A. A 3-year experience with laparoscopic gastric banding for obesity. Surg Endosc. 2000;14(6):532-536.

7. Cherian PT, Goussous G, Ashori F, Sigurdsson A. Band erosion after laparoscopic gastric banding: a retrospective analysis of 865 patients over 5 years. Surg Endosc. 2010; Feb 23. [Epub ahead of print].

8. O’Brien PE, Sawyer SM, Laurie C, et al. Laparoscopic adjustable gastric banding in severely obese adolescents: a randomized trial. JAMA. 2010;303(6):519-526.

9. Wittgrove AC, Clark GW. Laparoscopic gastric bypass, Roux-en-Y- 500 patients: technique and results, with 3-60 month follow-up. Obes Surg. 2000;10(3):233-239.

10. Coon D, Gusenoff JA, Kannan N, El Khoudary SR, Naghshineh N, Rubin JP. Body mass and surgical complications in the post-bariatric reconstructive patient: analysis of 511 cases. Ann Surg. 2009;249(3): 397-401.

11. Shermak MA, Chang D, Magnuson TH, Schweitzer MA. An outcomes analysis of patients undergoing body contouring surgery after massive weight loss. Plast Reconstr Surg. 2006;118(4):1026-1031.

12. Arthurs ZM, Cuadrado D, Sohn V, et al. Post-bariatric panniculectomy: pre-panniculectomy body mass index impacts the complication profile. Am J Surg. 2007;193(5):567-570.

13. Nemerofsky RB, Oliak DA, Capella JF. Body lift: an account of 200 consecutive cases in the massive weight loss patient. Plast Reconstr Surg. 2006;117(2):414-430.

14. Flancbaum L, Belsley S. Factors affecting morbidity and mortality of Roux-en-Y gastric bypass for clinically severe obesity: an analysis of 1,000 consecutive open cases by a single surgeon. J Gastrointest Surg. 2007;11(4):500-507.

15. Shermak MA. Hernia repair and abdominoplasty in gastric bypass patients. Plast Reconstr Surg. 2006;117(4):1145-1150.

16. Gusenoff JA, Coon D, Rubin JP. Brachioplasty and concomitant procedures after massive weight loss: a statistical analysis from a prospective registry. Plast Reconstr Surg. 2008;122(2):595-603. 
17. Rubin JP, Gusenoff JA, Coon D. Dermal suspension and parenchymal reshaping mastopexy after massive weight loss: statistical analysis with concomitant procedures from a prospective registry. Plast Reconstr Surg. 2009;123(3):782-789.

18. Losken A, Holtz DJ. Versatility of the superomedial pedicle in managing the massive weight loss breast: the rotation-advancement technique. Plast Reconstr Surg. 2007;120(4):1060-1068.

19. Bruschi S, Datta G, Bocchiotti MA, Boriani F, Obbialero FD, Fraccalvieri M. Limb contouring after massive weight loss: functional rather than aesthetic improvement. Obes Surg. 2009;19(4):407-411.

20. Ellabban MG, Hart NB. Body contouring by combined abdominoplasty and medial vertical thigh reduction: experience of 14 cases. Br J Plast Surg. 2004;57(3):222-227.
21. Shermak MA, Rotellini-Coltvet LA, Chang D. Seroma development following body contouring surgery for massive weight loss: patient risk factors and treatment strategies. Plast Reconstr Surg. 2008;122(1):280-288.

22. Buchwald H, Avidor Y, Braunwald E, et al. Bariatric surgery: a systematic review and meta-analysis. JAMA. 2004;292(14):1724-1737.

23. Pratt GM, Learn CA, Hughes GD, Clark BL, Warthen M, Pories W. Demographics and outcomes at American Society for Metabolic and Bariatric Surgery Centers of Excellence. Surg Endosc. 2009;23(4): 795-799. Epub 2009 Jan 30.
Open Access Surgery

\section{Publish your work in this journal}

Open Access Surgery is an international, peer-reviewed, open access journal that focuses on all aspects of surgical procedures and interventions. Patient care around the peri-operative period and patient outcomes post surgery are key topics. All grades of surgery from minor cosmetic interventions to major surgical procedures are covered. Novel techniques

Submit your manuscript here: http://www.dovepress.com/open-access-surgery-journal

\section{Dovepress}

and the utilization of new instruments and materials, including implants and prostheses that optimize outcomes constitute major areas of interest. The manuscript management system is completely online and includes a very quick and fair peer-review system. Visit http://www.dovepress.com/ testimonials.php to read real quotes from published authors. 